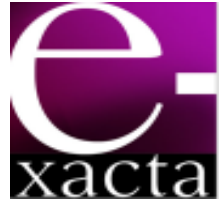

ISSN: 1984-3151

\title{
ESTUDO DE INTERFERÊNCIAS NA ALIMENTAÇÃO ELÉTRICA DE MOTORES DE INDUÇÃO POR INVERSORES DE FREQUÊNCIA
}

\author{
STUDY OF INTERFERENCE IN POWER SUPLLY FOR INDUCTION \\ MOTORS BY VARIABLE FREQUENCY DRIVERS
}

\author{
1 Engenheiro Eletricista. Centro Universitário de Belo \\ Horizonte - UniBH, 2012. Votorantim Siderurgia. Betim, \\ MG. alosousa@gmail.com \\ 2 Graduando em Engenharia Elétrica. UniBH, 2012. Jornal \\ Hoje em Dia. Belo Horizonte, MG. \\ marcosmoraisdasilva@hotmail.com \\ 3 Doutor em Engenharia Elétrica. PPGEE / UFMG, 2011 / \\ Professor do Departamento de Engenharia de Eletrônica \\ UFMG. Belo Horizonte, MG. iap@ufmg.br
}

André Luis de Oliveira e Sousa'; Marcos Morais da Silva ${ }^{2}$; Igor Amariz Pires ${ }^{3}$

Recebido em: 23/05/2012 - Aprovado em: 30/06/2012 - Disponibilizado em: 30/07/2012

\begin{abstract}
RESUMO: O presente trabalho realizou um estudo exploratório onde se apresenta os diversos fatores nos quais os motores de indução são submetidos ao serem acionados por inversores de frequência. O objetivo foi abordar aspectos técnicos que regem as aplicações de inversores com os motores de indução de gaiola. Assim, discutir as influências dos inversores sobre as características do motor, a reflexão de tensão, ruído de modo comum, risetime, etc, além da interferência da distância de instalação entre o inversor de frequência e o motor elétrico como agravante destes fatores. Também são apresentadas soluções que fabricantes de motores elétricos, inversores de freqüência, fornecedores de fios e materiais isolantes têm buscado com o objetivo de obter boas interações entre o motor elétrico e o inversor de frequência, evitando perturbações não só para o sistema em si, mas também para os sistemas adjacentes. Diante do tema abordado, define-se que os fatores apresentados podem interferir diretamente na vida útil do motor ou gerar perturbações em sistemas elétricos instalados próximos ao acionamento via inversor de frequência. Cabe dispor que como nos últimos anos somente um acionamento conquistou a confiança das indústrias no que diz respeito à substituição dos motores de corrente contínua, muitas empresas que fornecem o acionamento, não estão preparadas tecnicamente para especificar corretamente o conjunto, e, portanto, não conseguem evitar as interferências geradas conforme demonstrado no corpo deste trabalho.
\end{abstract}

PALAVRAS-CHAVE: Inversor de Frequência. Motor Elétrico de Indução. Isolação do Motor.

ABSTRACT: First of all, this work went into an exploratory study which shows the variety of factors that the induction motors are submitted for being driven by frequency inverters. It's target was to address the leading technical aspects of the induction motors cage, and also, to discuss the influences of the inverters about the motor characteristics such as reflection voltage, common mode of noise, rise-time, and so on. On the top of it, this work have had a deep study about the interferences from installation and the distance between the frequency inverter and electric motor such as the aggravation of these factors for both the inverter-motor system and for electrical systems. They are magnetically linked to this system by a bunch of wires and cables. Also, some solutions about electric motor's manufacturers are also showed. Inverters, wire's suppliers and insulation materials have sought in order to get a great interaction between the electric motor and frequency inverter to avoid disturbing the system. Not only for themselves but also for the adjacent systems. Once we had faced it's subject, we can define that the factors here presented can directly interfere with the engine's life or lead it into problems with the electrical systems, if installed next to the drive by a frequency inverter. As it is a kind of trigger that only had got the confidence of the industries recently, regarding to the replacement of DC motors, many companies that provide it, are not technically ready to correctly specify the whole set, in order avoid the interference generated as it was well showed in this work. 
KEYWORDS: Variable Frequency Drivers. Induction Eletric Motor. Isolation of Motor.

\section{INTRODUÇÃO}

Os acionamentos eletrônicos de velocidade variável abrangem um grupo muito grande de aplicações industriais. Cabe salientar que os eletrodomésticos já vêm incorporando esses acionamentos, como por exemplo, as máquinas lava-roupas. Por outro lado, os setores de transporte, o comercial e os de sistemas de potência também estão utilizando os acionamentos de velocidade variável como forma de conservação de energia. As aplicações dos acionamentos eletrônicos estão se tornando cada vez maiores e pode-se dizer que atualmente são irreversíveis. Desse modo, há uma maior exigência por parte dos fabricantes de conversores estáticos de frequência e de máquinas elétricas, bem como uma compreensão maior dos elementos que compõem este sistema.

Antes do surgimento do primeiro dispositivo semicondutor de potência (tiristor) no final da década de 60 , os acionamentos das máquinas com variação de velocidade só eram possíveis através de variadores mecânicos (polias, redutores), variadores hidráulicos ou através de máquinas de corrente contínua. No entanto, esta última em menor escala devido à dificuldade de prover um controle adequado da mesma nesta época conforme Karavasilis (2008). As principais características deste tipo de acionamento são a simplicidade, a facilidade de controle e a possibilidade de acionamento em potências elevadas. No entanto, sabe-se que o uso dos motores CC apresenta uma série de limitações, sendo as principais delas:

- O comutador e escovas requerem manutenção;

- Devido à comutação, as máquinas CC não podem ser utilizadas em áreas de risco como minas, indústria química, petroleira e outras;
- A relação de potência por peso ou o volume de um motor CC é maior que a de um motor CA, considerando, principalmente, os motores de indução do tipo gaiola de esquilo.

A necessidade de ter maior consciência para a conservação energética, fez com que a eletrônica de potência juntamente com outras áreas da ciência, tivesse uma evolução surpreendente. As duas últimas décadas presenciaram o aperfeiçoamento de novos semicondutores de potência com controle de condução e bloqueio, além de possibilidade de trabalho em tensões e correntes elevadas, o que abriu boas perspectivas para o controle de velocidade em motores de corrente alternada.

Contudo, serão apresentadas propostas de soluções tecnológicas a serem utilizadas em instalações para minimizar as interferências nas alimentações dos motores elétricos, a partir das redes elétricas, com o objetivo final de obter a melhor performace do equipamento.

\section{FUNDAMENTOS DE INFLUÊNCIA DO INVERSOR SOBRE O SISTEMA DE ISOLAÇÃO}

Há alguns anos, para se obter um controle preciso de velocidade, eram utilizados motores de corrente continua, entretanto, isso acarretava diversos problemas, como custo do motor e necessidade de retificação da tensão de fornecimento para alimentar o motor, segundo Franchi (2008). Com o avanço da eletrônica de potência e a necessidade de aumento produtivo e redução de custo, surgiu a automação, ainda em fase inicial no Brasil.

Segundo o mesmo autor, o sinal de saída de tensão e corrente em um inversor de frequência é não senoidal 
e possui diversas frequências harmônicas, além da elevada frequência de chaveamento dos módulos IGBT's que vem do Inglês Insulated Gate Bipolar Transistor ou, em Português Transistor Bipolar de Porta Isolada. Esses fatores são causadores do aparecimento de alguns fenômenos que podem danificar o motor, o inversor de frequência, o cabo de alimentação e, até mesmo, gerar interferências em equipamentos ligados próximos a esse sistema. Isto exige, por parte dos fabricantes de inversores de frequência e de máquinas elétricas, maior compreensão dos elementos que compõem este sistema, assim como, a interação entre eles.

De acordo com Karavasilis (2008), os inversores de frequência ou conversores estáticos de frequência, baseiam-se na conversão da tensão da rede alternada senoidal na entrada do dispositivo, em tensão contínua e, finalmente, convertem esta última, em uma tensão de amplitude e frequência variáveis. Inerentemente ao projeto básico de um inversor de frequência, tem-se na entrada o bloco retificador, o circuito intermediário composto de um banco de capacitores eletrolíticos e finalmente o bloco inversor. Na FIG.1 tem-se o esquema básico de um inversor de frequência.

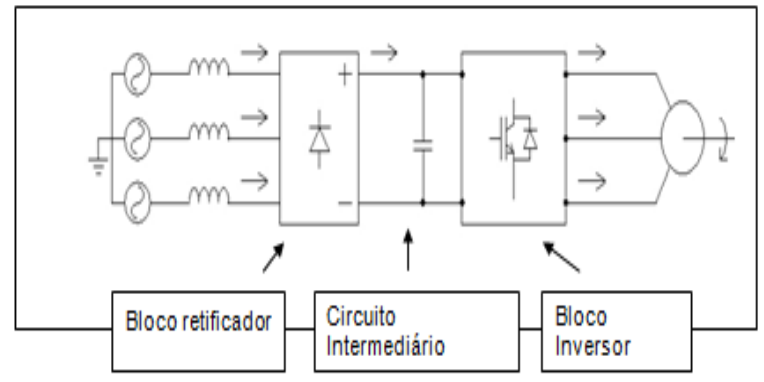

Figura 1 - Circuito Básico de um Inversor de Frequência

\subsection{MOTOR ELÉTRICO DE INDUÇÃO}

O motor assíncrono, conforme Uliana (2008), é constituído basicamente pelos seguintes elementos:
- um circuito magnético estático, constituído por chapas ferromagnéticas empilhadas e isoladas entre si, ao qual se dá o nome de estator; por bobinas localizadas em cavidades abertas no estator e alimentadas pela rede de corrente alternada;

- um rotor constituído por um núcleo ferromagnético, também laminado, sobre o qual se encontra um enrolamento ou um conjunto de condutores paralelos, nos quais são induzidas correntes provocadas pela corrente alternada das bobinas do estator.

O mesmo autor ressalta que, o rotor é apoiado num veio, que por sua vez transmite à carga a energia mecânica produzida. $\mathrm{O}$ entreferro (distância entre o rotor e o estator) é bastante reduzido, de forma a reduzir a corrente em vazio e, portanto as perdas, mas também para aumentar o fator de potência em vazio.

A FIG.2 demonstra a projeção dos diversos elementos o motor assíncrono de rotor em gaiola de esquilo.

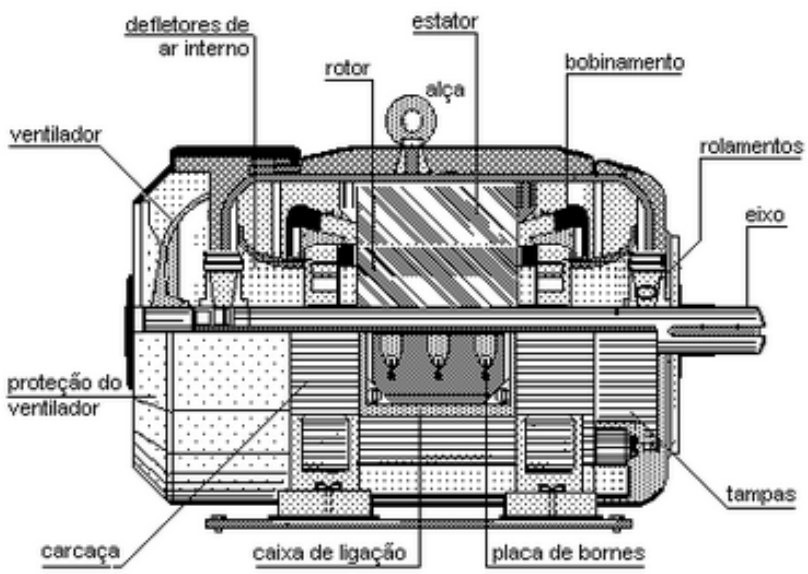

Figura 2 - Projeção de um motor de indução Fonte - WEG, 2006 


\subsection{INFLUÊNCIA DO TEMPO DE SUBIDA (RISE}

\section{TIME)}

Segundo Karavasilis (2008), certa quantidade de tempo é requerida para que a tensão nos terminais de saída do inversor transite de seu valor mínimo até o seu valor máximo, esses pulsos de tensão, após percorrerem o cabo de alimentação entre o conversor de frequência e o motor, atingem os terminais do motor. Como a impedância do motor elétrico é muito superior àquela apresentada pelo cabo, a velocidade de propagação dos pulsos de tensão é atenuada nos enrolamentos do motor. Ainda devido a essa grande diferença de impedância entre o motor elétrico e o cabo, a tensão nos terminais do motor poderá ser somada a uma componente de reflexão, sendo que o comprimento do cabo é fator determinante nas características desta reflexão. Desta forma, o pulso de tensão ao atingir os terminais do motor, faz com que a(s) primeira(s) espira(s) da primeira bobina de uma dada fase, fique $(\mathrm{m})$ submetida(s) a um alto valor de tensão. Com isso, o rise time tem influência direta no tempo de vida útil do sistema isolante do motor, ou seja, quanto menor o tempo de crescimento do pulso (rise time), maior será a taxa de variação da tensão (dV/dt) e maior a diferença de potencial (ddp) originada entre espiras, consequentemente, a degradação do sistema de isolamento do motor será mais rápida.

O mesmo autor ressalta que, espiras adjacentes das primeiras bobinas de uma dada fase, ficam submetidas a uma elevada ddp em função dos pulsos de tensão que chegam aos terminais do motor elétrico de indução, conforme é demonstrado na FIG.3.

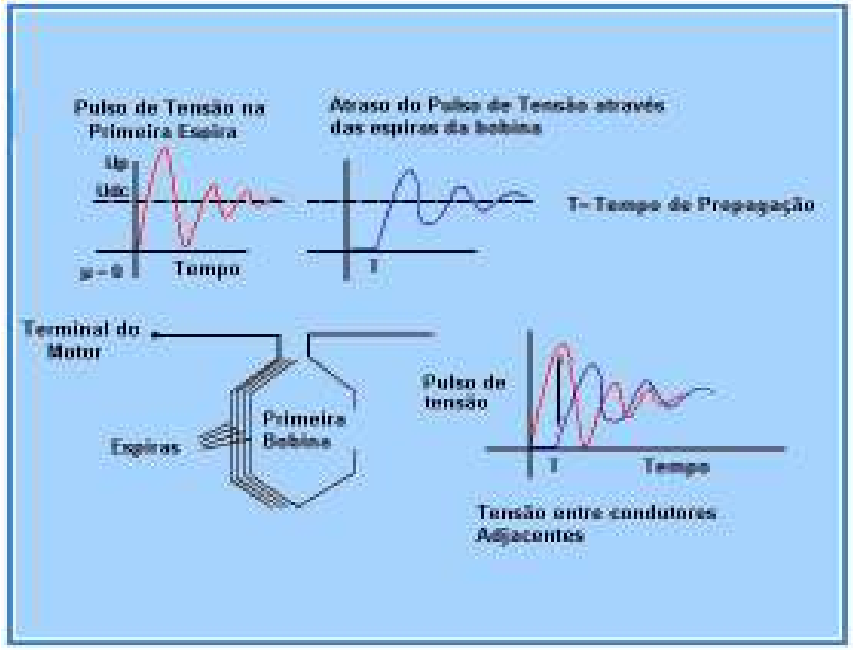

Figura 3 - Propagação de um pulso de tensão nos enrolamentos de um motor Fonte - KARAVASALIS, 2008, p.16.

\subsection{INFLUÊNCIA DO CABO DE ALIMENTAÇÃO}

Para o sucesso de uma instalação de um acionamento de motor através de inversor de frequência, como afirma Karavasilis (2008), que tem como objetivo o desempenho e durabilidade, é muito importante considerar o cabo entre o inversor e o motor como sendo parte do sistema. Os cabos de comprimento elevado, conforme o guia de aplicação da norma NEMA para sistemas de acionamento de velocidade variável, aumentam o valor do overshoot (pico de tensão) nos terminais do motor. Com os modernos IGBTs, os overshoots começam a aparecer a partir de, aproximadamente, $3 \mathrm{~m}$ de cabo.

Ainda assim, o mesmo autor afirma que, o cabo pode ser considerado uma linha de transmissão, sendo uma longa matriz de seções de indutâncias e capacitâncias conectadas em serie/paralelo, conforme é demonstrado na FIG.4. 


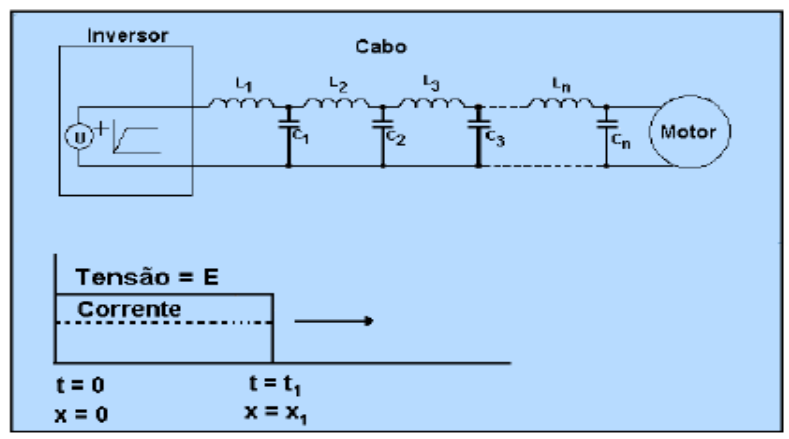

Figura 4 - Indutâncias e capacitâncias distribuídas do cabo

Fonte - KARAVASALIS, 2008, p.22

\subsection{RUÍDO EM MODO COMUM}

Castro (2012) afirma que o ruído de modo comum é o responsável por alguns erros em malhas de 4 a $40 \mathrm{~mA}$ e erros de comunicação devicenet RS232/RS485 entre outros. Eles acontecem a cada chaveamento dos IGBT's e pode ser visto na FIG.5.

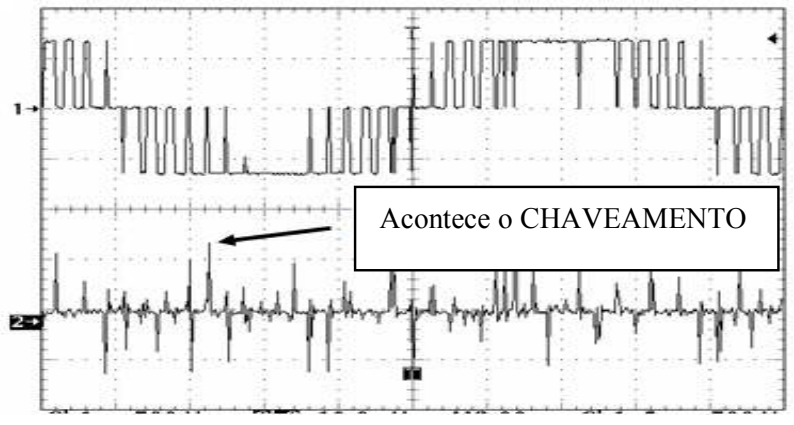

Figura 5 - Ruído modo comum Fonte-CASTRO, 2012, p.18.

Para o mesmo autor, existem dois tipos de ruídos, que são: os ruídos irradiados onde o cabo de força funciona como antena e, o ruído conduzido, onde sinais elétricos circulam pelos cabos e malhas de terra.

O ruído de modo comum é proveniente da alta frequência existente no chaveamento dos IGBT's, o que produz correntes parasitas através de capacitâncias parasitas, que são pequenas capacitâncias do sistema, que em $60 \mathrm{~Hz}$ não são significativas, mas em altas frequências passam a perturbar o funcionamento do circuito.

\subsection{INFLUÊNCIA DA FREQUÊNCIA DE PULSAÇÃO DE TENSÃO}

A freqüência dos pulsos de tensão dos inversores de frequência pode alcançar atualmente $20 \mathrm{kHz}$, com a real tendência de elevação deste valor, em função da rápida evolução que a eletrônica de potência tem apresentado. A condição torna-se ainda mais inóspita para o sistema de isolação, uma vez que, para cada pulso de tensão emitido pelo conversor de frequência, as sobretensões nos terminais do motor poderão ocorrer no início e no fim do mesmo, conforme apresentado pela FIG.6.

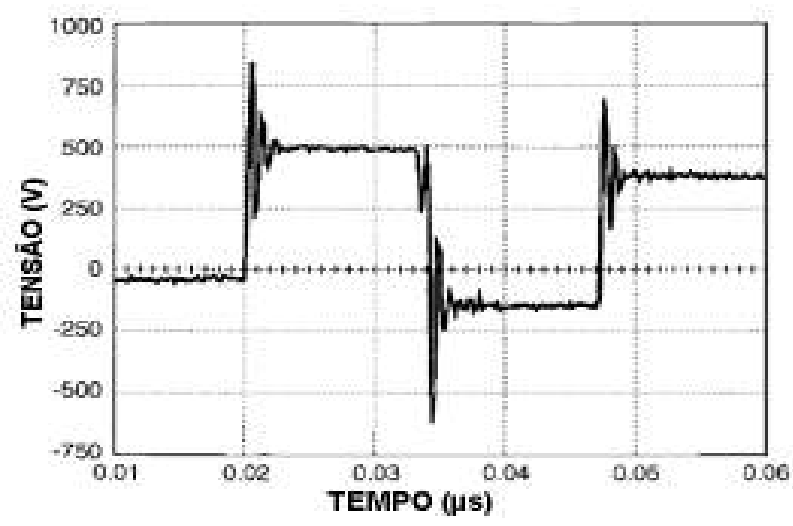

Figura 6 - Tensão fase-fase nos terminais do motor Fonte - KARAVASALIS, 2008, 32 p.

Para o mesmo autor, é importante ainda salientar que, a integridade do isolante torna-se comprometida somente na ocorrência de cada pulso de tensão, ou seja, o intervalo de tempo entre cada pulso não contribui para a deterioração do isolante, uma vez que, o mesmo não estará sendo submetido ao esforço provocado pelo pulso. Assim, quanto maior a freqüência de pulsação e consequentemente maior o número de pulsos de tensão num dado intervalo de tempo, mais rápido ocorrerá a falha no sistema de isolação. 


\subsection{INFLUÊNCIA DA FREQUÊNCIA DE PULSAÇÃO DE TENSÃO}

Segundo Karavasilis (2008), o sistema de controle dos inversores PWM faz o ajuste de velocidade dos motores (tensão e frequência), através da variação da largura dos pulsos e do tempo entre estes pulsos. As sobretensões estarão potencialmente na pior condição para ocorrerem (maiores amplitudes), quando o tempo entre os pulsos consecutivos for o mínimo. Esta condição pode ocorrer principalmente durante os picos da onda fundamental da tensão de saída e durante condições transitórias, tais como, a aceleração e a desaceleração do motor elétrico. Se o tempo entre pulsos consecutivos (tpc) for menor do que 3 vezes, o tempo do período de ressonância do cabo (tc), o tempo para o trajeto através do cabo, conforme ilustrado na FIG.7, ocorrerá uma superposição do primeiro sinal (1) com o segundo sinal (2), os quais são pulsos consecutivos. Com esta superposição, a amplitude resultante será maior, nos terminais do motor, pois, poderá ter o sinal do segundo pulso, somado com a sua própria reflexão mais o sinal do primeiro pulso, também somado à sua própria reflexão. Para evitar este efeito de superposição dos picos das ondas de dois pulsos consecutivos, a regra deve ser considerada a Eq.1.

$$
t p c>(3 \times t c)
$$

onde:

tpc= Tempo entre pulsos consecutivos, $[\mu \mathrm{s}]$. tc= Período de ressonância do cabo alimentador entre inversor e motor, [ $\mu \mathrm{s}]$.

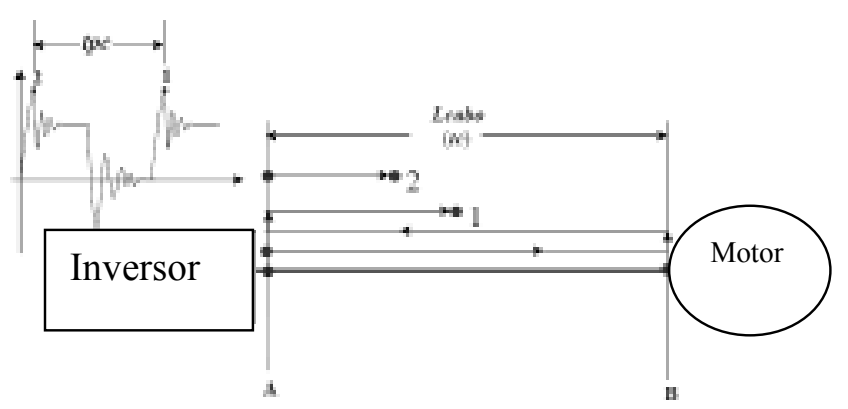

Figura 7 - Tempo entre pulsos consecutivos e a superposição de sinais Fonte - KARAVASALIS, 2008, p.32.

\subsection{LARGURA DE PULSOS}

Conforme mostrado na FIG.8, a maior tensão de pico $\left(U_{\text {PICO }}\right)$, devido à ddp que poderá surgir na bobina, entre as espiras, em função do tempo tb, pode ser formada pelos picos de dois fenômenos distintos: a superposição de sinais, devido à reflexão da onda de tensão e o efeito da tensão de auto-indução, devido à comutação (abertura) da chave (transistor). Tendo em vista o grande amortecimento produzido pelo enrolamento, pode-se dizer que, este efeito combinado ocorre basicamente sobre a primeira bobina, ou seja, a bobina de entrada do sinal. Ainda sobre a primeira bobina, pode-se dizer que, o fenômeno ocorre sobre as primeiras espiras, devendose ter em conta ainda os efeitos aleatórios das posições espaciais de cada espira num enrolamento randômico.

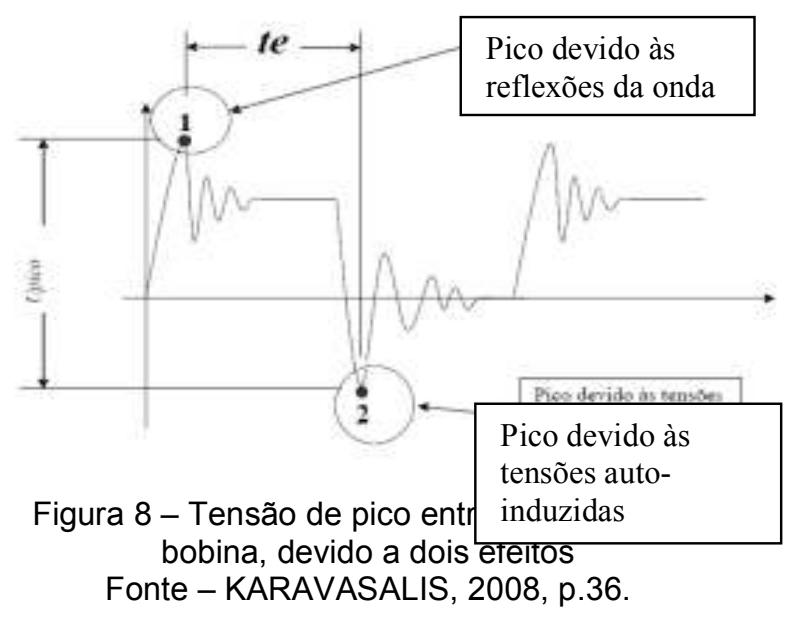


Karavasalis (2008) afirma que, para evitar a ocorrência dos efeitos simultaneamente (reflexão e auto-indução) sobre uma mesma bobina, o que pode originar uma altíssima tensão entre espiras, deve-se ter tempos tb, para o trajeto das ondas de tensão através da bobina, sempre menores do que os tempos te, entre os picos dos dois fenômenos. Portanto, para evitar o efeito simultâneo da superposição de sinais sobre uma mesma bobina, a regra deve ser seguida na Eq.2.

$$
\text { te }>t b
$$

onde:

te $=$ Tempo decorrido entre o pico de tensão devido à reflexão de onda e o pico de tensão devido ao efeito de auto-indução na interrupção da corrente, [ $\mu s]$.

tb = Período de tempo necessário para os pulsos percorrerem o caminho através de uma bobina, [ $\mu \mathrm{s}]$.

Assim sendo, Karavasilis (2008) afirma que para a formação do sinal da tensão de saída, o qual corresponde à harmônica fundamental na freqüência nominal do motor, as larguras dos pulsos individuais formadores deste sinal, não podem ser muito pequenas, para evitar, consequentemente, valores muito pequenos para o tempo te.

\subsection{ESFORÇO ELÉTRICO NOS SISTEMAS DE} ISOLAÇÃO DOS ENROLAMENTOS DAS MÁQUINAS

Karavasilis (2008) afirma que quando o enrolamento de uma máquina elétrica é submetido a impulsos de tensão, com elevada taxa de crescimento no tempo (dV/dt) e com acentuada magnitude, tem-se um esforço de alta tensão nas regiões entre condutores de fases diferentes (fase-fase), entre um condutor e o terra (fase-terra) e, entre espiras adjacentes (entre espiras) conforme apresentado pelas FIG.9 e FIG.10.
O mesmo autor afirma que, com isto, as taxas de crescimento da onda de tensão relativamente às distâncias de isolamento, ou seja, os gradientes de potencial nas diversas partes do sistema isolante assumem uma proporção muito grande, tornando-se decisivos, visto que os tempos para ocorrer a degradação dos materiais isolantes e consequentemente a vida útil dependem destes gradientes.

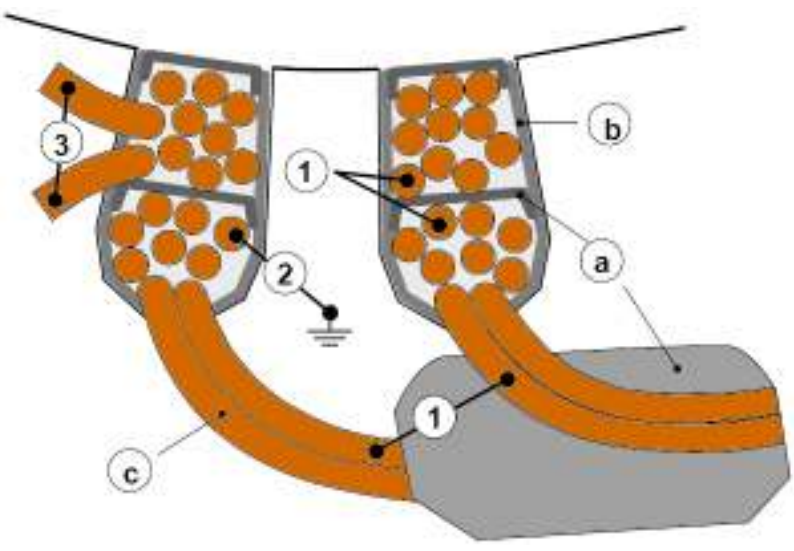

Figura 9 - Projeto com enrolamento randômico Fonte - KARAVASALIS, 2008, p.38.

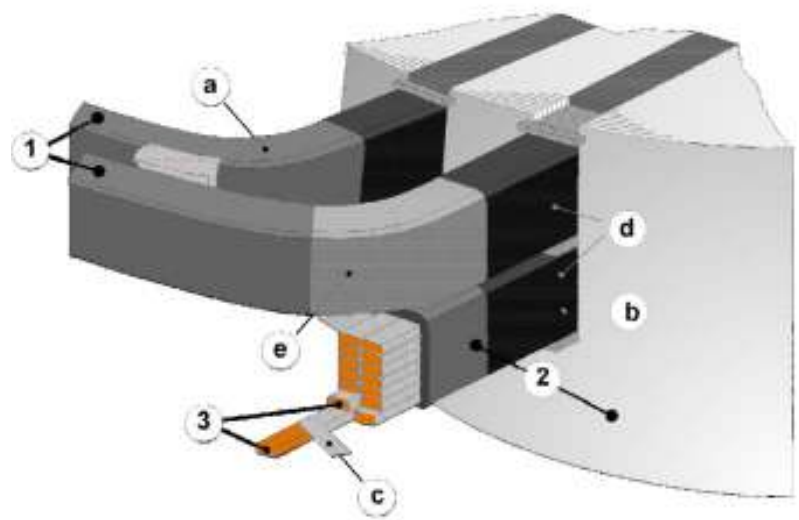

Figura 10 - Projeto com bobinas pré-formadas Fonte - KARAVASALIS, 2008, p.38.

\section{Metodologia}

Os procedimentos metodológicos foram iniciados com uma pesquisa bibliográfica sobre o princípio de 
funcionamento dos inversores de frequência e de motores elétricos de indução em corrente alternada, onde são empregados.

O segundo passo, consistiu em mostrar as estratégias de eliminar as interferências na alimentação dos motores através de inversores de frequência, mostrando os desenvolvimentos de sistemas isolantes para o motor elétrico. Assim, esforços em pesquisas têm se intensificado por parte de fabricantes de motores elétricos, de materiais isolantes e de fios utilizados nos motores, com o objetivo de desenvolver sistemas isolantes mais robustos e aptos à utilização com inversores de frequência.

Ao fim, também foram apresentadas algumas técnicas comuns utilizadas na instalação entre o inversor de frequência e o motor elétrico, com o objetivo de modificar as formas de onda de tensão apresentada aos terminais do motor.

O trabalho se classifica como descritivo, segundo Karavasilis (2008), pois tem por objetivo de especificar conceitos sobre inversores de frequência em verificação da alimentação de motores elétricos de indução.

\section{Resultados}

Trabalho intenso tem sido feito na melhoria de confecção de fios. Um novo tipo de fio foi projetado com isolação resistente às descargas parciais e aos surtos de tensão os quais podem estar acima do valor inicial de tensão para a ocorrência das descargas. A isolação apresenta alta estabilidade térmica, dissipando rapidamente os pontos de aquecimento, para prevenir os danos causados pelo aquecimento do dielétrico resultante dos pulsos com elevado rise time e alta frequência. $A$ isolação ainda possui funções de dissipação de carga, para eliminar as cargas espaciais que se originam durante os pulsos com rápidos rise time.

A FIG.11 apresenta os resultados obtidos com o novo fio resistente a pulsos desenvolvido por seu fabricante. Os resultados são respectivamente comparados com os atuais fios com isolamento padrão. O nível de ozônio, a temperatura e a circulação de ar são controlados durante a realização dos ensaios, pois tais variáveis impactam diretamente no valor limiar de tensão em que as descargas parciais irão ocorrer. O desenvolvimento de fios com sistemas de isolação apresentando melhores características de suportabilidade é vital para a integridade do motor elétrico quando acionado por conversor de frequência.

Há também a importância de melhorar a robustez do isolamento de motores acionados através de inversores de freqüência, isto tem sido reconhecido e o tema foi estudado por fabricantes de motores. Critérios especiais de projeto e práticas de fabricação foram desenvolvidos para motores com tal aplicação. A FIG.12 apresenta a comparação do envelhecimento térmico e de tensão para um isolamento com esmalte e outro com mica, ambos com o mesmo dielétrico de pico de tensão. O primeiro demonstrativo do gráfico apresenta os números sobre a resistência térmica e de tensão (Esmalte em Azul, Mica em Vermelho) para $\mathrm{CA}, 60 \mathrm{~Hz}$ e, o segundo, sobre PWM, $14 \mathrm{kHz}$. 


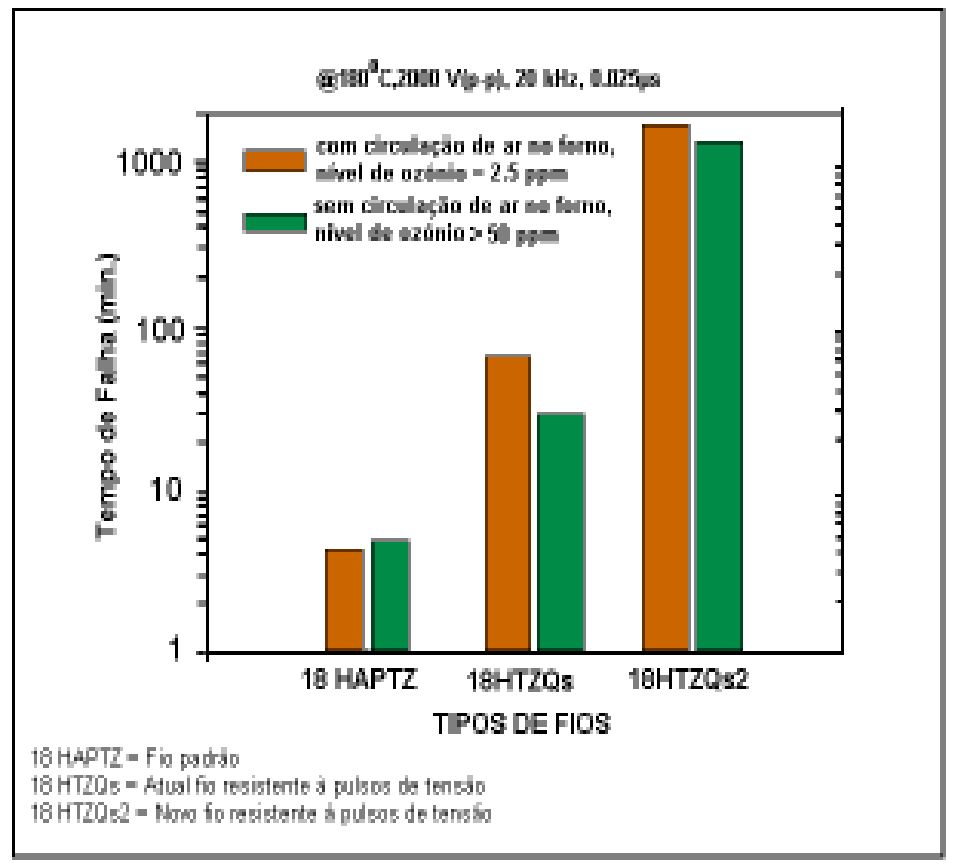

Figura 11 - Comparação da performance de resistência à pulsos de fios a $180^{\circ} \mathrm{C}, 20 \mathrm{kHz}, 2 \mathrm{kV}(\mathrm{p}-\mathrm{p})$ e $25 \mathrm{~ns}$ Fonte - KARAVASALIS, 2008, p.49.

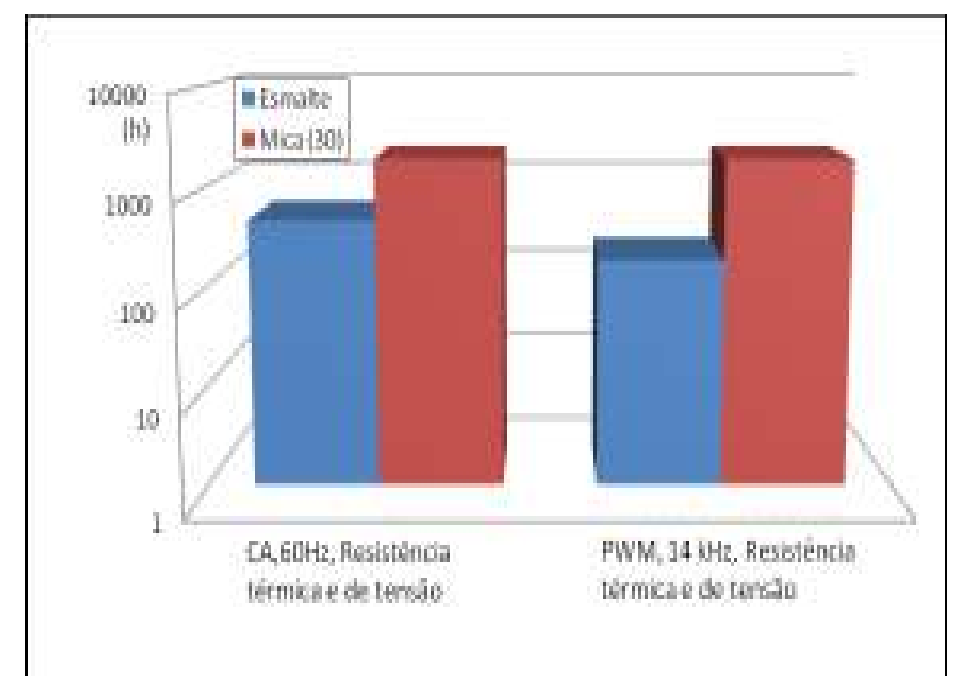

Figura 12 - Comparação da resistência térmica e de tensão de espiras isoladas com esmalte e outras com mica, ambas numa situação sob a freqüência nominal da rede de alimentação e noutra sob uma tensão PWM (14 kHz).

Testes realizados a $3 \mathrm{kV}$ (pico) e $155^{\circ} \mathrm{C}$ Fonte - KARAVASALIS, 2008, p.51.

Mesmo com as melhorias nas condições de isolação e considerações de projeto direcionadas ao motor elétrico em algumas aplicações, não é possível atingir as características de suportabilidade exigidas pela instalação. Assim, torna-se necessária a implementação de técnicas que proporcionem a modificação da tensão nos terminais do motor. Estas técnicas são essencialmente as colocações de mecanismos entre o motor e o inversor para limitar a taxa de crescimento do pulso, reduzir o coeficiente de reflexão e por meio disso, reduzir o nível da tensão de pico. Estas técnicas podem ser resumidas em: Reatores de Saída; Filtros dV/dt de Saída; Filtros senoidais e Filtros RC. 
Os reatores de saída, especialmente projetados, os quais podem acomodar a forma de onda PWM sem apresentar aquecimentos indevidos e podem também fornecer os valores de indutância necessários para o espectro de freqüência exigido pela aplicação. Tais reatores foram utilizados para reduzir o $\mathrm{dV} / \mathrm{dt}$ e o pico de tensão.

Normalmente o reator de saída é montado dentro do compartimento do painel metálico do inversor de frequência, com a consequente necessidade de espaço adicional, custo e redução da eficiência do sistema. Ao adicionar um reator na extremidade do

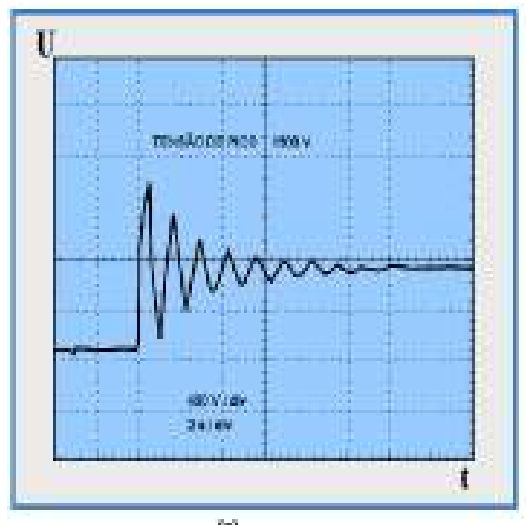

(a) cabo que está conectado ao inversor de frequência, a impedância de surto do cabo será modificada. Como a impedância de surto do cabo é normalmente menor que a do motor, aumentando tal valor artificialmente, o coeficiente de reflexão será menor o qual reduzirá a magnitude da onda refletida.

Para a forma de onda apresentada pela FIG.13, a adição do reator estendeu o tempo de crescimento do pulso em aproximadamente $5 \mu$ s e reduziu o pico de tensão para $792 \mathrm{~V}$, o qual é aceitável para a isolação da maioria dos motores padrões.

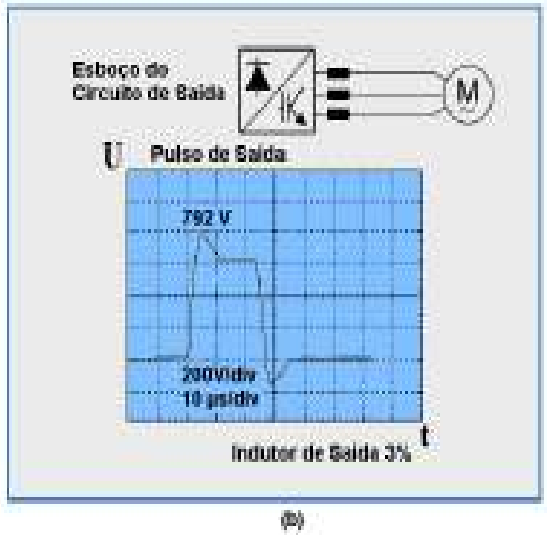

Figura 13 - (a) Sinal de entrada e (b) Sinal de saída obtido com a utilização de um reator de saída Fonte - KARAVASALIS, 2008, p.59.

Na FIG.14 o pico de tensão é reduzido à $684 \mathrm{~V}$ com um $\mathrm{dV} / \mathrm{dt}$ de $40 \mathrm{~V} / \mu \mathrm{s}$. Tais filtros permitiram o uso da maioria dos motores sem problemas e são então recomendados se os dados do motor são

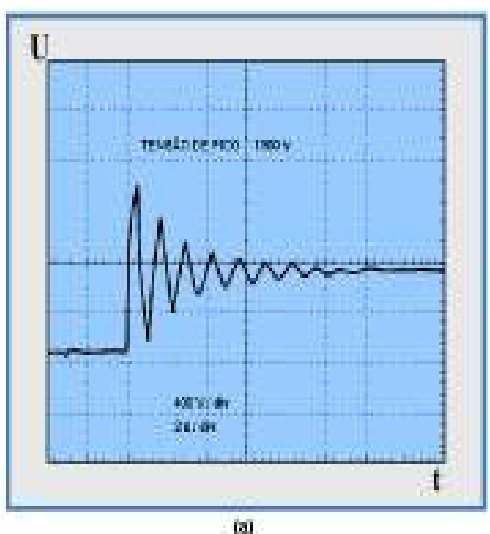

desconhecidos, como no caso de motores já existentes, mas que a instalação está sendo modernizada com a instalação de inversores de frequência.

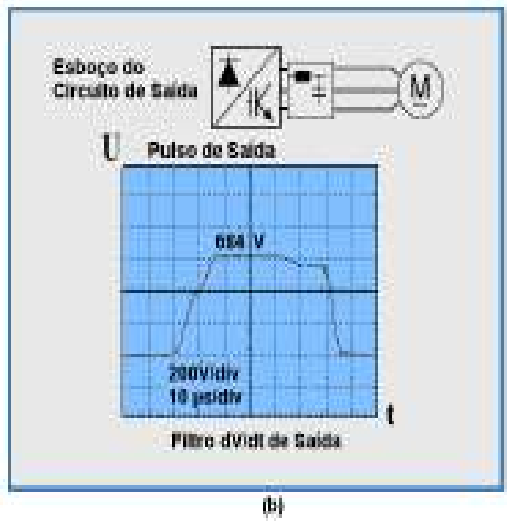

Figura 14 - (a) Sinal de entrada e (b) Sinal de saída obtido com a utilização de um dv/dt de saída Fonte - KARAVASALIS, 2008, p.60. 
Os filtros senoidais consistem basicamente da combinação de um reator e um capacitor formando um filtro LC passa baixa. O conceito é que o filtro possui uma frequência de ressonância de aproximadamente 1 a $1,5 \mathrm{kHz}$ e as frequências maiores que aquelas serão absorvidas pelo filtro e não apresentadas ao motor. Obviamente é importante que a frequência de chaveamento do conversor seja colocada próxima de $1 \mathrm{kHz}$, ou seja, maior que a frequência de ressonância para prevenir o excesso de correntes filtradas. Assim, o filtro passa baixa permite que as correntes de alta frequência sejam bloqueadas. Isto faz com que as formas de onda nos terminais do motor se tornem aproximadamente senoidais. As formas de onda da tensão e da corrente são, para um ciclo, como apresentado pela FIG.15. Estes tipos de filtros são os mais caros, no entanto, eles apresentam as seguintes vantagens:

- Reduzem o ruído do motor;

- Reduzem as perdas do motor;

- Simplifica a certificação de motores para áreas classificadas;

- Permite o uso de motores com longos cabos até o inversor de frequência.

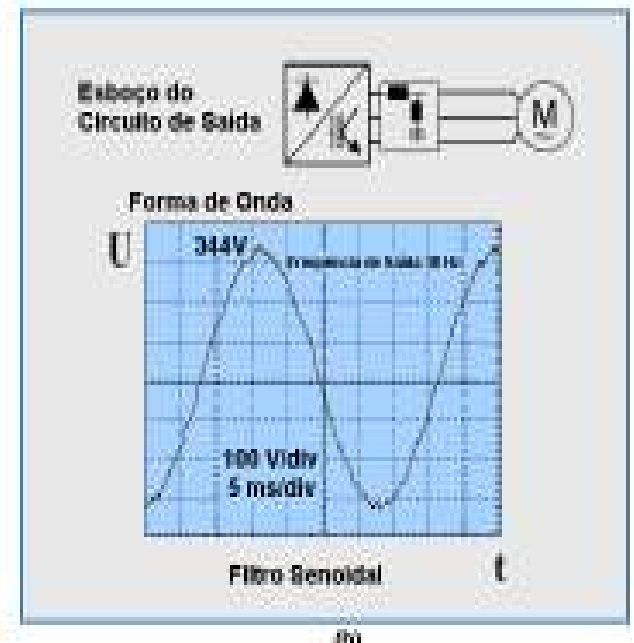

(ii)

Figura 15 - (a) Sinal de entrada e (b) Sinal de saída obtido com a utilização de um filtro senoidal Fonte - KARAVASALIS, 2008, p.61.

\subsection{MEDIÇõES COM DIVERSAS METRAGENS DE CONDUTORES ELÉTRICOS}

Nas FIG.16, 17, 18, 19 e 20, foram realizadas medições da tensão na saída com diversos comprimentos de cabos para verificar a influência dos ruídos na alimentação dos motores acionados por um inversor de frequência modelo CFW09 da WEG. 


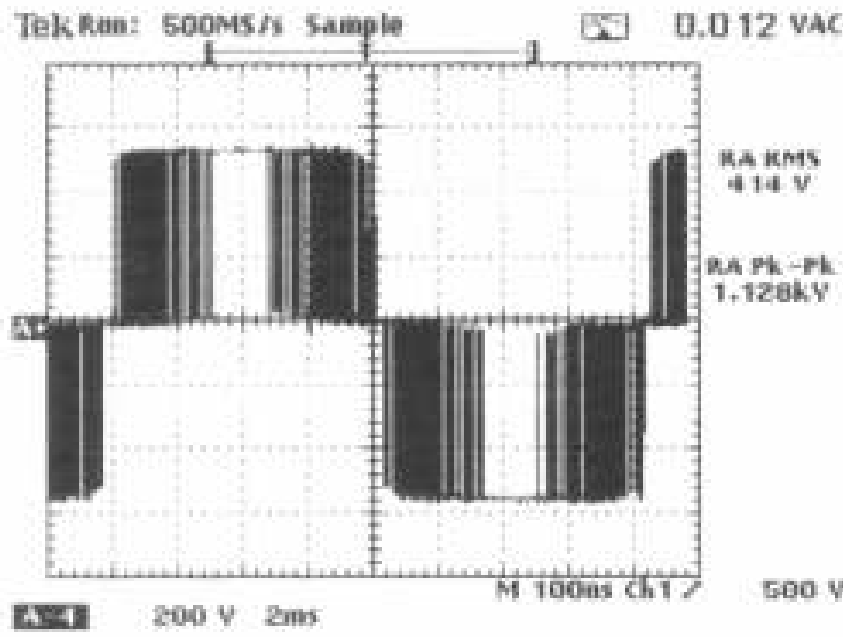

Figura 16 - Tensão nos terminais de saída do inversor - sem cabo

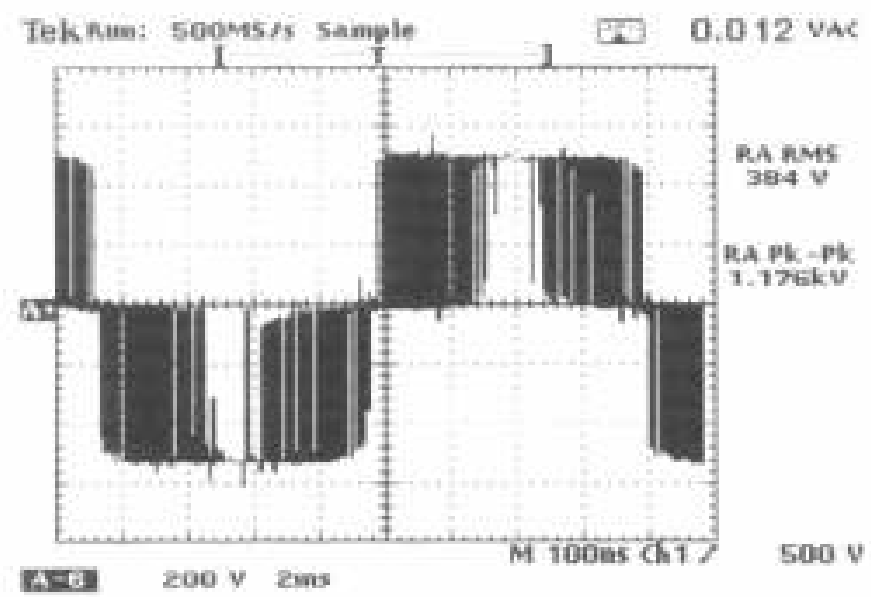

Figura 17 - Tensão nos terminais de saída do inversor - 1,5m de cabo

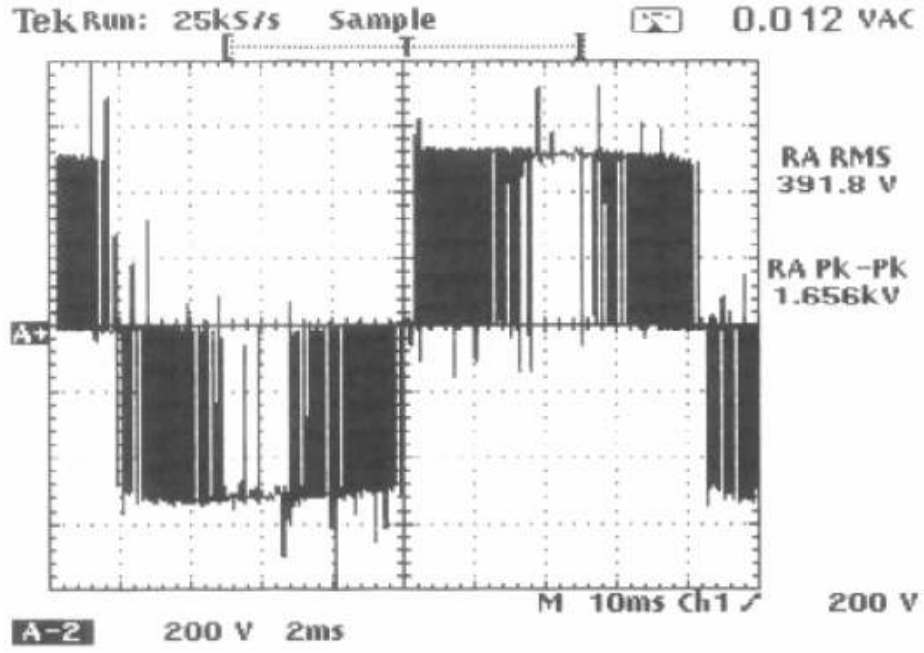

Figura 18 - Tensão nos terminais de saída do inversor - 15m de cabo 


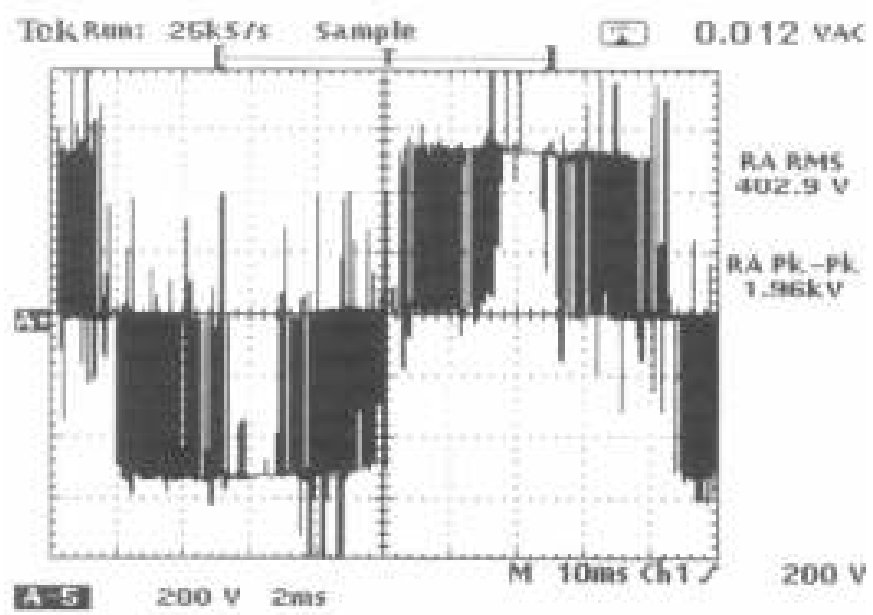

Figura 19 - Tensão nos terminais de saída do inversor - 34m cabo

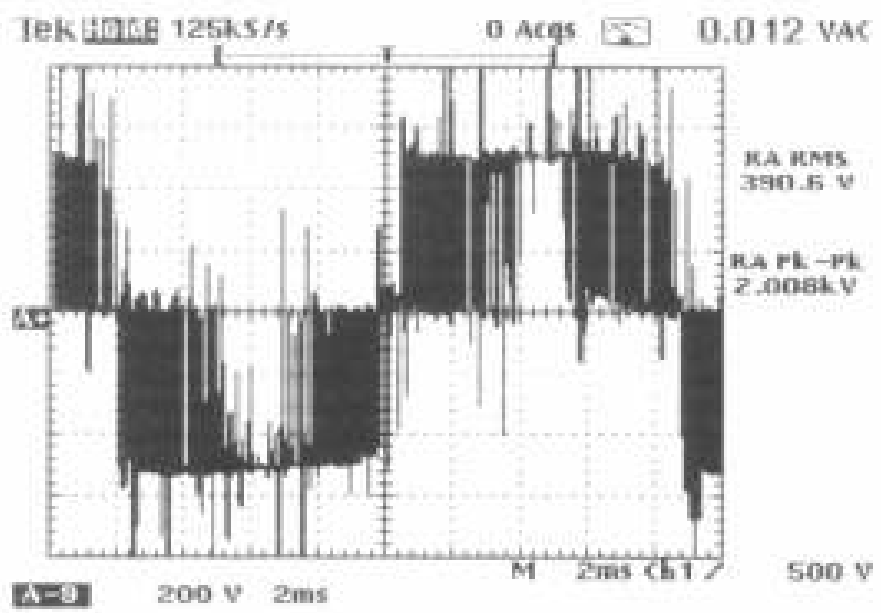

Figura 20 - Tensão nos terminais de saída do inversor - 54m cabo

Para o comprimento de cabo de $42 \mathrm{~m}$, foram apresentados exemplos ilustrando resultados que puderam ser obtidos para a forma de onda de tensão nos terminais do motor ao implementar-se cada uma das técnicas.

Há também problemas que podem ocorrer com as possíveis causas como sobretensão da rede, baixa impedância da rede, link CC ou IGBTs em curto ou problemas de fiação.

Para estes casos deve-se verificar se os varistores de entrada estão queimados, se estiverem significa que houve sobretensão. Se não estiverem, então a instalação (impedância da rede, fiação) deverá ser verificada.

Os picos de corrente na entrada são limitados pela impedância da rede, pela reatância de rede ou pelo indutor do link CC. Os picos de corrente elevados são prejudiciais tanto para o retificador quanto para os capacitores do link CC. As formas de onda da FIG. 21 mostram a corrente de entrada em um inversor de fabricação WEG de corrente nominal de 142A, alimentado em tensão de $380 \mathrm{~V}$, trabalhando com $100 \%, 50 \%$ e $30 \%$ de carga, sem reatância, com $2 \%$ e com $4 \%$ de reatância. 

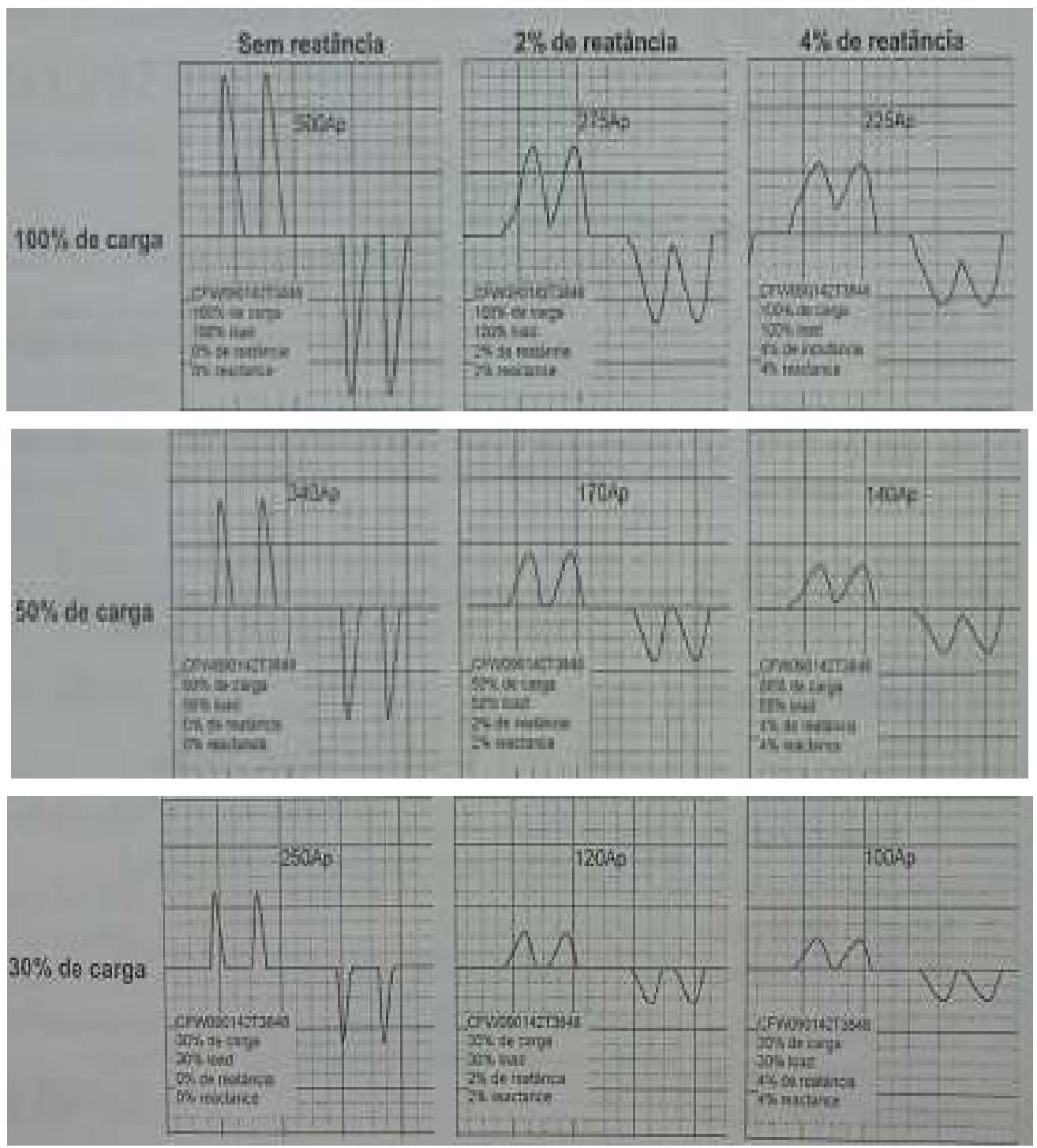

Figura 21 - Testes com cargas e reatâncias escalonadas Fonte - WEG, 2009, p.23.

\section{ConClusão}

Pelo presente trabalho, pode-se observar que existem diversos fatores comumente deixados de lado por fabricantes de painéis elétricos com acionamento via inversor de frequência. Conforme demonstrado neste trabalho, tais fatores podem interferir diretamente na vida útil do motor ou até mesmo gerar perturbações em sistemas elétricos próximos à instalação do acionamento via inversor de frequência.

Por se tratar de um tipo de acionamento relativamente novo e que só nos últimos anos conquistou a confiança das indústrias, muitas empresas fornecedoras de soluções para as diversas aplicações industriais não estão preparadas tecnicamente para especificar todos os equipamentos que formam o conjunto (Motor elétrico, inversor de freqüência, cabos elétricos, filtros, etc). Isso gera prejuízos para empresa contratante, já que o investimento acaba não sendo recompensado da forma que deveria.

Percebeu-se que devido à insuficiência técnica destas empresas fornecedoras de solução, os fabricantes dos equipamentos que formam o conjunto inversor de 
frequência-motor elétrico, cabo elétrico de alimentação do motor e motor elétrico, não têm medido esforços para minimizar os efeitos aqui estudados, com o intuito de que, a má especificação técnica do conjunto feita pelas empresas fornecedoras de soluções, não gere tanto prejuízo para a empresa contratante. docente do curso de Engenharia Elétrica do UniBH pela confiança, incentivos e presteza no auxílio às atividades e discussões sobre o tema deste trabalho.

Também à empresa LOG Automação e Sistemas por fornecer materiais, catálogos e manuais dos equipamentos, laboratório e componentes para desenvolvimento deste.

\section{AgRADECIMENTOS}

Os autores agradecem aos Professores e corpo

\section{REFERÊNCIAS}

CASTRO, Gleston F. Inversor de freqüência, conceitos e técnicas de aplicação. Local: TECNAUT, 2012, 58 p. II. Disponível em: http://www.tecnaut.com.br/old/utilidades/Tecnicas $\% 20$ e\%20cuidados.pdf. Acesso em: 10 jan. 2012.

FRANCHI, Claiton. Inversores de Frequência: Teoria e Aplicações. 2a ed. São Paulo: Érica, 2011. 192 p.

KARAVASILIS, Renato. Motores Acionados por Conversores de Frequência. Florianópolis / SC: UFSC, 2008. 148p.

ULIANA, Jorge. Comandos e Motores Elétricos. Criciúma / SC, 2008. 14 p.
WEG. Guia de aplicações de inversores de frequência Jaraguá do Sul / SC: Weg, 2002. 192 p. il.

WEG. Manual do Inversor de Frequência CFW-09. Jaraguá do Sul / SC: Weg, 2009. 12 p. il.

WEG. Motores de indução alimentados por inversores de frequência PWM. Jaraguá do Sul / SC: Weg, 2006. 35 p. il. 